\title{
Postgenomic technologies in practical forestry: development of DNA markers and population genetic databases for timber origin identification, genetic monitoring, breeding and other applications
}

\author{
Krutovsky K.V. \\ Laboratory of Population Genetics, Vavilov Institute of General Genetics, RAS, Moscow, Russia \\ Laboratory of Forest Genomics, Genome Research and Education Center, Siberian Federal University, \\ Krasnoyarsk, Russia \\ Department of Forest Genetics and Forest Tree Breeding, Georg-August University of Göttingen, Germany \\ Department of Ecosystem Science and Management, Texas A\&M University, College Station, USA \\ e-mail: konstantin.krutovsky@forst.uni-goettingen.de
}

The forest genetics, tree improvement and protection can greatly benefit from complete genome sequence data made recently available for several major conifer species. They allow to identify and annotate genes, other functional elements (sRNA, transcription factors, regulatory elements, etc.), and genetic networks that control adaptation and disease resistance. They can be used to develop highly informative genetic markers that can be applied in population genetic studies to create database of barcodes for individual populations to fight illegal timber harvest and trade. They are very much needed for development of genome-wide genetic markers for association studies for linking genetic variation (SNPs, alleles, haplotypes, and genotypes) with environmental factors, adaptive traits and phenotypes for better understanding genetic control of agronomically and economically important traits. They can be also used to develop genome-wide genetic markers for genomic-assisted selection to breed for better adapted, stress resistant and climate change resilient trees with desirable quality ecological and economic traits. Finally, whole genome sequences allow to integrate proteomics, transcriptomics, and metabolomics and provide reference genomes for resequencing. One of the most important practical applications of genomics in forestry, which will be presented in detail is development of highly polymorphic and informative DNA markers for several very important boreal forest species in Eurasia, Siberian larch (Larix sibirica Ledeb.), Siberian stone pine (Pinus sibirica Du Tour), and Scots pine (Pinus sylvestris L.), based on the whole genome data obtained in the "Genomics of the Key Boreal Forest Conifer Species and their Major Phytopathogens in the Russian Federation" project funded by the Government of the Russian Federation (grant No. 14.Y26.31.0004). 